\title{
ASSESSMENT PLATFORM OF WATER AND ENERGY EFFICIENCY IN COLLECTIVE IRRIGATION SYSTEMS
}

\author{
André Antunes, Diogo Felicíssimo, Bruno Ferreira and Nelson Carriço \\ Instituto Politécnico de Setúbal, Campus do IPS - Estefanilha, 2910-761 Setúbal, Portugal
}

\begin{abstract}
Southern European countries do not have regular precipitation to assure the agricultural water needs, since precipitation occurs scarcely from April to October. As such, irrigation is essential for agriculture and is only possible with the existence of hydraulic infrastructures for storage and water conveyance. In Portugal, these hydraulic infrastructures are mostly public, forming collective irrigation systems, and managed by Irrigation Associations.

The efficiency of irrigation should be assured through a set of guidelines and procedures promoting a more efficient use of water and energy. Furthermore, in Portugal there are currently no performance assessment system developed to evaluate the water and energy efficiency in CIS. The current practice for the assessment of water and energy efficiency is a set of reactive and unintegrated actions. As such, the gathered information is not usually organized and does not follow systematic procedures. Additionally, appropriate indicators for water and energy loss diagnostic are not defined, thus making it impossible to compare efficiencies between CIS.

In this context, the AGIR R\&D project aiming the establishment of a performance assessment system for water and energy efficiency assessment in CIS was developed. One of the outcomes of the project was the development of a user-friendly platform for the CIS management utilities to assess its water and energy efficiencies.

This paper presents the platform AGIR, its functionalities and main components. The platform was designed allowing systematic and uniform data introduction, for usage by various decision support modules.
\end{abstract}

\section{KEYWORDS}

Water Efficiency, Energy Efficiency, Collective Irrigation Systems, Assessment Tool, Information System

\section{INTRODUCTION}

In Portugal, water usage and energy efficiency are some of the main concerns of Irrigation Associations (IA) managing collective irrigation systems (CIS) (Carriço, N. et al., 2018; DGADR, 2017), and is a priority of recent public policies, mostly due to increasing regulatory pressure from Common Agricultural Policy (CAP), and achieved through the promotion of economic and environmental efficiency.

Currently, however, there is no objective-oriented assessment system nor a set of appropriate performance indicators allowing IAs to quantify water and energy efficiencies in their conveyance and distribution networks, as well as to support the decision-making process.

\section{The AGiR project}

The AGIR research project is born in this context. Named "AGIR - Water Use and Energy Efficiencies' Assessment in Collective Irrigation Systems", is coordinated by the Portuguese National Irrigators Federation (FENAREG) and funded by PDR2020 in Operation 1.0.1 - Operational Groups.

This project has as its main objectives the establishment of a performance indicators system for water and energy efficiency assessment in CIS and the development of a set of usable and user friendly tools to be used by CIS management utilities. These developments should provide ways for assessment and identification of the inefficiencies within the systems, as well as to support the selection of interventions for improving the water use and energy efficiency' and energy recollection. One of AGIR research project outputs is a software platform for water and energy balance calculation and a performance assessment system, allowing CIS and agricultures to evaluate the efficiency of water use and energy consumption. 


\section{The software platform}

The software development project uses an agile methodology. The project has the support of the research team and end-user partners, following the development process through work meetings for discussion and evaluation. As such, the requirement analysis has been an iterative process. System design has been updated as needed to reflect the needs presented by the research team and end-user partners. The methodology used in the development of the project allows easier change management and adaptation for change integration, since project changes can be rather frequent and relevant during the development process. As such, the process of analysis and design for the current stage of the platform was an iterative process, where an initial requirement analysis allowed the establishment of some fundamental actions for the platform.

IA and other water resources managing utilities need a tool for water and energy efficiency assessment of CIS. This tool must be generic enough to accommodate the distinct system infrastructures and measurements needed for the several expected calculations.

As described above, the assessment of water use and energy consumption efficiency is not a common practice among CIS and irrigators. As such, the software platform development faced some challenges, namely, data insertion (i.e., considering that most CIS don't currently use geographical information systems) and visualization (i.e., requirements for simple and intuitive dashboards and menus).

This paper focus on the development of the modules and tools, as well as the implementation of functionalities in the software platform. This platform allows the uniform and systematic data insertion by the IA, for later use by the several integrated decision-making support modules. These modules include the water and energy balance' calculations, allowing hydric efficiency assessment (Loureiro, D. et al, 2019; Cunha, H. et al, 2019), and energy efficiency assessment (Cunha, H., 2018), as well as a performance assessment system.

In addition, a farmer login functionality is provided, allowing the qualitative hydric efficiency evaluation of the irrigation network, obtained through terrain analysis, irrigation mechanics and meteorological conditions (regarding the farmer context) in a simple and intuitive way. So, the platform is designed for two distinct users, IA and farmers. The user type is defined during the registration process and determines the available platform areas and functionalities.

The platform developed during the AGIR project presents itself as a novel alternative for the system performance assessment, allowing IA to perform organized and dynamic calculation of the diverse system profile components, from network total length to more complex calculations (i.e. minimum operation volume in canals), that were previously done using spreadsheets.

The platform uses a logical hierarchy, imposing dependencies between system components (e.g. the infrastructure component is inaccessible before the selection of a given CIS). In this way, the user is lead in providing the needed data for the water and energy balance's and performance assessment system. In the end, performance indicators are produced, thus allowing the IA to assess the CIS performance during a time period.

\section{SYSTEM DESIGN}

The process of system analysis and design for the current stage of the platform was an iterative process. An initial analysis, based on the research project requirements, allowed definition of the fundamental components and modules for the platform. System design has been updated as needed to reflect the needs presented by the research team and end-user partners.

\subsection{Platform Architecture}

Platform deployment is based on Docker. Docker technology is used in system deployment allowing greater development and maintenance flexibility. Two distinct Docker images are used. A first Docker image contains the MySQL Database Management System (DBMS) and system database. The second Docker image contains the environment and platform application. Communication between the two Docker images is established. The server was implemented using Node.js technology employing the JavaScript language. A set of web services and a frontend web application are provided. The client-server system architecture can be visualized in Figure 1. 


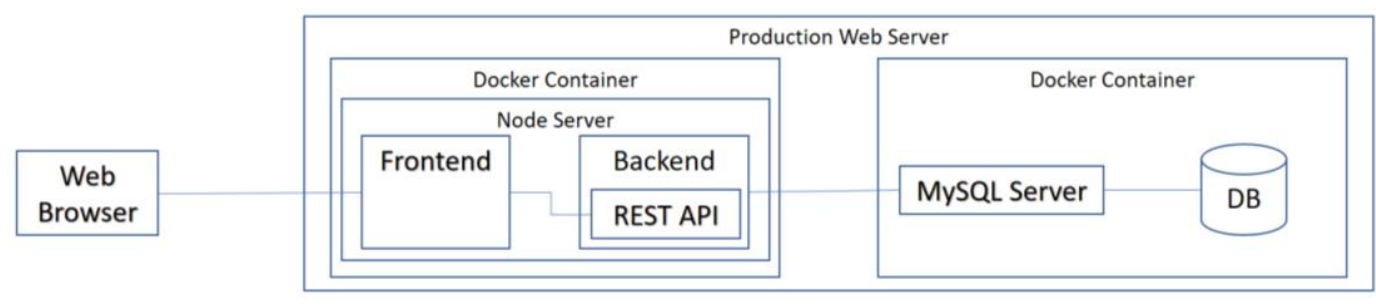

Figure 1. System Architecture

Users access the system with a session login, using the internet and a browser. In order to be able to initiate a session, users must first register in the platform. The application starting page provides users with registration and login functionalities. The platform has been designed for two distinct actors, IA and farmers. The user type is defined during the registration process and determines the accessible application areas and available system functionalities.

Each IA can manage one or more CIS. Each CIS has its own infrastructure, consisting in catchments, canals and canal elements, pipes and pipe elements, stations and reservoirs. Each CIS managed by the IA can have years of analysis set up. For each year of analysis, the IA can add irrigation seasons. The irrigation season allows the IA to associate measurements to the CIS infrastructural elements. These measurements are afterwards used in the calculation of water and energy balances and assessment system.

Farmers are independent from any IA and can use the system for the evaluation of the water use efficiency of their irrigation systems. However, they can associate their applications to a certain IA, allowing the later access to the farmer's water use efficiency data. That allows the IA to query those efficiency evaluations through the web application.

Figure 2 shows the diagram of the logical hierarchy described.

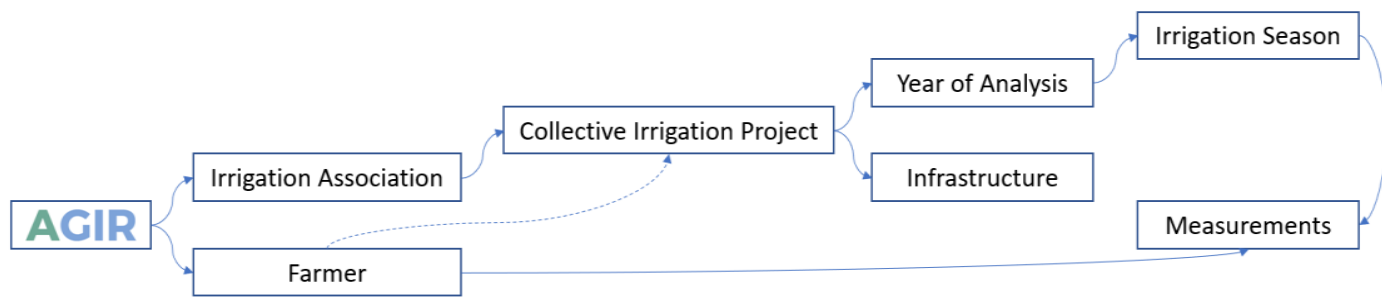

Figure 2. Logical hierarchy

\subsection{Data Model}

For the development of this platform, a data model was designed from scratch (Figure 3), reflecting the data needs for the end-user partners when modelling their systems. The DBMS used is MySQL.

Since the water and energy balances and the performance assessment system have distinct data calculation requirements, the platform has 4 main sections, namely: authentication, farmer efficiency, infrastructure and measurements. Infrastructural data refer to structural elements, relatively stable in time, such as intakes, canals, canal elements, pipes and pipe elements, pumps and pumping stations and intermediate reservoirs. Measurements refer to data measured through time during a specific Irrigation Season, from a distinct Year of Analysis. These measurements are used afterwards for water and energy balance' and the performance evaluation system. 


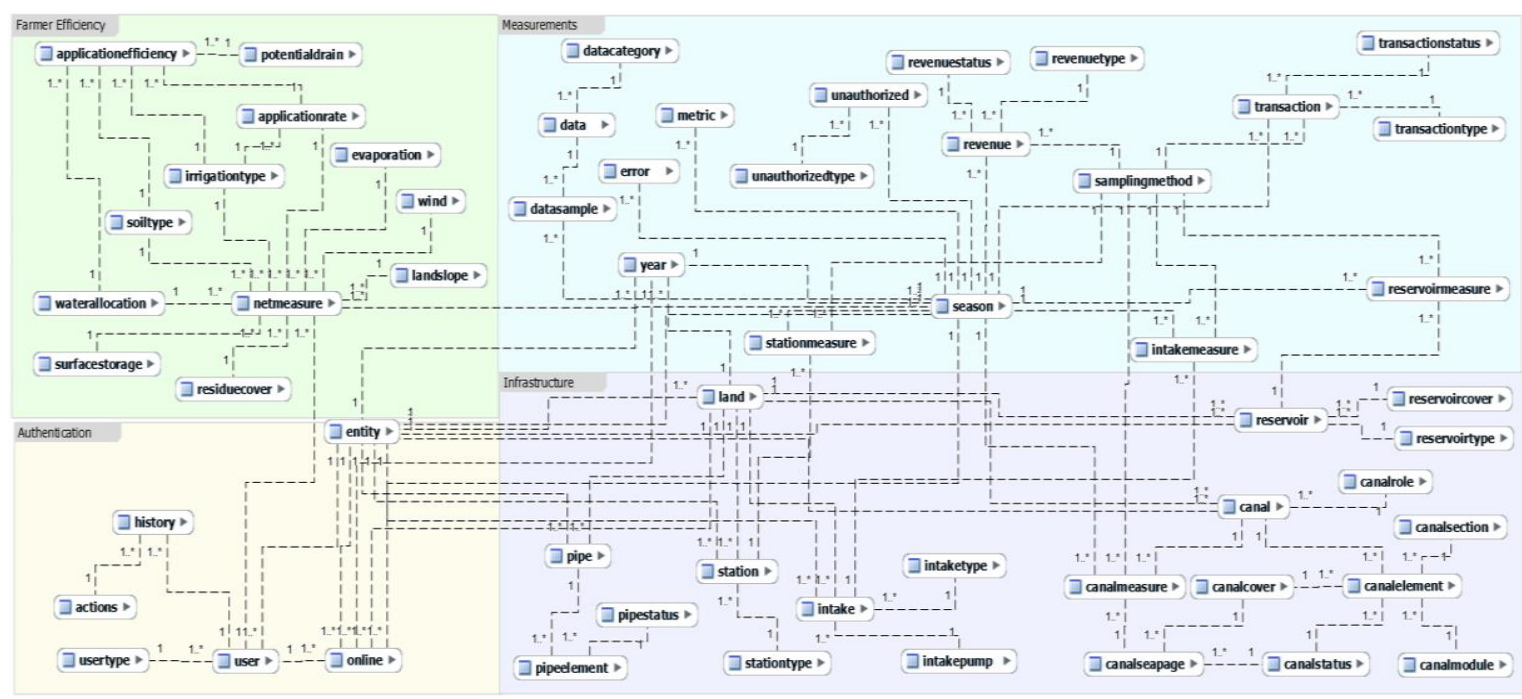

Figure 3. AGIR Data Model

\subsection{Modules}

The system follows a modular approach and is composed by a set of four main modules: authentication, infrastructure, water balance and farmer's irrigation network modules. Modules for energy balance and the performance assessment system are under research and will be implemented and integrated in the platform. The following section present the infrastructure module and the novel water balance and farmer's irrigation network modules.

\subsubsection{Infrastructure Module}

The infrastructure module allows the introduction of infrastructural elements. Each CIS has a specific infrastructure that needs to be inserted. Infrastructural elements include intakes, canal and canal element, pipe and pipe element, pumping stations and reservoir.

An infrastructure full report is available, listing all CIS infrastructural elements.

\subsubsection{Water Balance Module}

The water balance module allows the calculation of water balance (H. Cunha et al, 2019), for an irrigation season (in a specific year of analysis), of a specific CIS.

Measurements associated with infrastructural elements such as intakes, canals and reservoirs are used in the calculation of water balance (e.g., canal cross-section). Other considered measurements are authorized and unauthorized consumption, as well as metering inaccuracies.

Several complex calculations are carried for the different infrastructures. As an example, for each reservoir a water balance is carried and for each canal section the surface subject to evaporation is calculated. Due to the complexity involved in the calculation of water balance, the developed module is novel a valuable tool the efficient management of water use in CIS.

\subsubsection{Farmer's Irrigation Network Module}

The farmer's irrigation network module allows the assessment of the water usage efficiency of their water applications. The calculation has several interdependent variables the user can set up with the resulting efficiency calculated and displayed in real time (Figure 4).

Variables used to obtain a final efficiency for the water usage include soil type, regarding hydraulic conductivity (light, medium, heavy or crust) and irrigation mechanism (sprinkler, center pivot or drip). Each irrigation mechanism has its own intrinsic property values such as wet area and flow. The calculation of the water application rate is based on the entered values. 
Land slope $(<2 \%, 2$ to $5 \%,>5 \%)$ and green cover (low, medium, high) values determine the surface storage quantity and potential runoff. The average wind intensity (low, medium, high) and evaporation (low, medium, high) are meteorological factors to taken into consideration directly into the water use efficiency. The irrigation rate (low, medium, high) is also required for the application efficiency calculation.

Application efficiency and apparent losses percentages are displayed as final calculation result. A chart with the applicable ranges for the resulting efficiency is displayed. The range associated to the resulting efficiency is highlighted, when applicable. Chart ranges vary according to soil type and irrigation mechanism' used. (Luz, P. B., 2002; Luz, P. B. \& Silva, L. L., 2004).

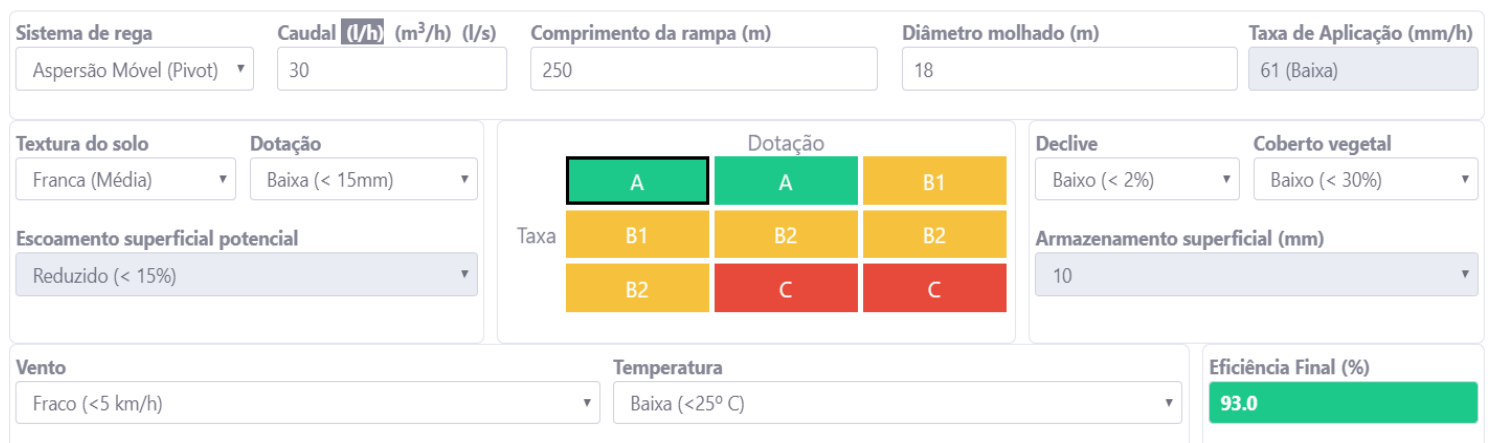

Figure 4. Farmer's Irrigation Network module

\section{CONCLUSION}

This paper presented an innovative software-platform to the data integration and assessment of water use in Collective Irrigation Systems. This platform presents itself as the first step in the development of a reference tool to support decision-making in Collective Irrigation Systems, enabling the improvement of the water usage and energy efficiencies. Future work includes the implementation of the remaining modules, such as, energy balance and performance evaluation system.

\section{ACKNOWLEDGEMENT}

The authors gratefully acknowledge all project AGIR partners that directly or indirectly contributed to the presented paper. This research was funded by PDR2020 grant number PDR2020-101-031878.

\section{REFERENCES}

Carriço , N. et al., 2018, Eficiência hídrica e energética em aproveitamentos hidroagrícolas. $14^{\circ}$ Congresso da Água.

Cunha, H., 2018. Balanços Hídrico e Energético em Aproveitamentos Hidroagrícolas - Uma nova abordagem para sistemas mistos. Instituto Superior Técnico - Universidade de Lisboa.

Cunha, H. et al, 2019. A comprehensive water balance methodology for collective irrigation systems. Agric. Water Manag., vol. 223, no. May, p. 105660.

DGADR, 2017. Sistema de Informação do Regadio. Autoridade Nacional do Regadio. [Online]. Available: http://sir.dgadr.gov.pt/stat. [Accessed: 21-Jan-2020].

Loureiro, D. et al, 2018, Proposta de balanços hídrico e energético para diagnóstico de perdas de água e de ineficiências energéticas em aproveitamentos hidroagrícolas. VII Congresso Nacional de Rega e Drenagem.

Luz, P. B., 2002, Sistematização da informação para apoio ao regadio no âmbito agro-ambiental. Avaliação do risco de escoamento superficial em rampas rotativas. Actas do $6^{\circ}$ Congresso da Água. APRH. Porto.

Luz, P. B. \& Silva, L. L., 2004, Avaliação de objectivos ambientais na rega por rampa rotativa. Aplicação de metodologias em solo mediterrâneo. Actas do $7^{\circ}$ Congresso da Água. APRH. Lisboa. 